\title{
EBV related cerebral lymphoma in a leukemia patient treated with alemtuzumab
}

\author{
B. van de Langerijt $\cdot$ J. K. Doorduijn • \\ K. H. Lam • M. J. van den Bent
}

Received: 19 August 2010/Revised: 14 November 2010/Accepted: 25 November 2010/Published online: 7 December 2010

(C) The Author(s) 2010. This article is published with open access at Springerlink.com

Dear Sirs,

A 56 year old man was diagnosed with B-chronic lymphocytic leukemia (B-CLL) in another hospital (RAI 4, BINET C, IGHV mutated; FISH: $59 \%$ of cells trisomy 12). Treatment was started with fludarabine, cyclophosphamide and alemtuzumab. The patient received pneumocystis jiroveci pneumonia prophylaxis and herpes prophylaxis with sulphamethoxazole-trimethoprim and valacyclovir. Evaluation after two cycles showed evidence of response. Screening for cytomegalovirus (CMV) and Epstein-Barr virus (EBV) antibodies using ELISA was repeatedly negative. After 2 months the patient developed memory loss and a sensory aphasia, and treatment including alemtuzumab was discontinued. A cerebral MRI scan revealed several lesions with some irregular and faint ring enhancement in the occipital and frontal lobe of the left hemisphere and the frontal lobe of

B. van de Langerijt · M. J. van den Bent $(\square)$

Department of Neuro-Oncology, Daniel den Hoed Cancer

Center/Erasmus University Hospital, PO Box 5201,

3008 AE Rotterdam, The Netherlands

e-mail: m.vandenbent@erasmusmc.nl

B. van de Langerijt

e-mail: b.vd.langerijt@elisabeth.nl

B. van de Langerijt

Department of Neurology, St Elisabeth Hospital,

Tilburg, The Netherlands

J. K. Doorduijn

Department of Hematology, Erasmus University Hospital

Rotterdam, Rotterdam, The Netherlands

e-mail: j.doorduijn@erasmusmc.nl

K. H. Lam

Department of Pathology, Erasmus University Hospital

Rotterdam, Rotterdam, The Netherlands

e-mail:k.lam@erasmusmc.nl the right hemisphere, with high signal intensity on $\mathrm{T} 2$ weighted images (Fig. 1). Treatment with amoxicillin and cefotaxime was started and the patient was referred to our hospital. Cerebrospinal fluid (CSF) showed 10 leucocytes (71\% lymphocytes) with an elevated total protein $(1,08 \mathrm{~g} / \mathrm{l})$, normal glucose and a monoclonal B-cell population consistent with the B-CLL (CD5, CD19 and CD23 positive). All CSF cultures for micro-organisms were negative. Two days after the last negative $\mathrm{IgG}$ and $\mathrm{IgM}$ screening for EBV, PCR for EBV in serum and CSF showed high numbers of viral copies, respectively, 5,01E5 and 1,31E4. A biopsy of the left parietooccipital lesion revealed a diffuse large B-cell lymphoma (DLBCL), positive for CD79a and CD23 and nuclear Pax-5. MIB-1 labeling was positive in $85 \%$ of the tumor cells. The nuclear EBV-encoded RNA stain (EBER) was strongly positive, fitting in with the development of an EBV-associated lymphoma. Despite high dose dexamethasone the patient deteriorated rapidly and he died 15 days after the initial MRI cerebrum. Autopsy was not performed.

Symptomatic CNS involvement in patients with B-CLL is an uncommon complication and generally limited to the meninges. Intracerebral localisations are exceedingly rare [4]. Although development of an aggressive large-cell lymphoma in patients with an underlying CLL occurs in $1-10 \%$ of patients, only six case reports on malignant transformation of CLL (or Richter's transformation) involving the brain parenchyma have been published [2].

Alemtuzumab (Campath-1H) is an anti-CD52 humanized monoclonal antibody [6]. It is indicated for poor prognosis CLL and the drug is being investigated in combination therapies for a variety of hematological malignancies and in multiple sclerosis. Because of its effects on $\mathrm{B}$ and $\mathrm{T}$ lymphocytes with prolonged $\mathrm{T}$-cell deficiency, the drug is highly immunosuppressive. Indeed, alemtuzumab is associated with a variety of opportunistic 
A

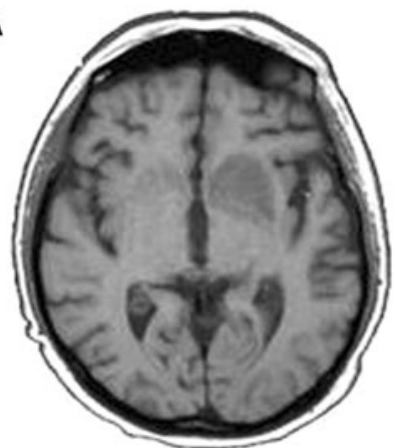

B

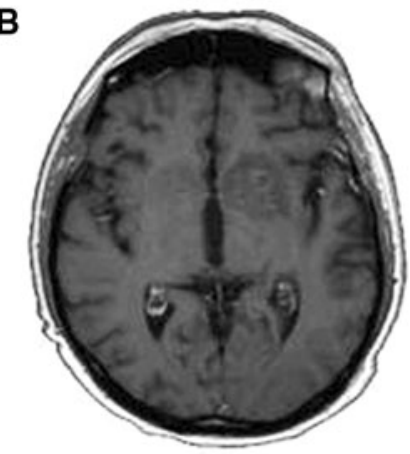

C
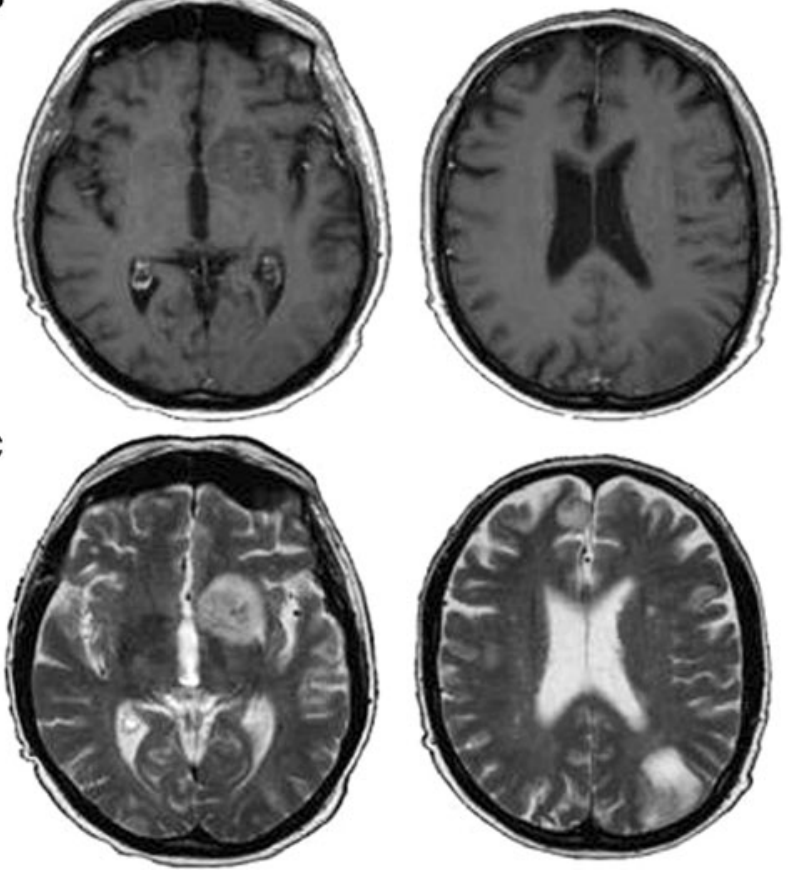

Fig. 1 Cerebral MRI Axial MRI images a-c showing lesions in the central and occipital regions of the left hemisphere and frontal region of the right hemisphere with low signal intensity on T1-weighted sequences (a), faint ring enhancement on T1-weighted sequences after gadolinium (b) and high signal intensity with subtle low signal intensity parts on $\mathrm{T} 2$ weighted sequences (c). The low signal intensity parts on T2 weighted sequences suggests a lymphoma, but the faint ring enhancement is not typical for this diagnosis

infections, especially CMV reactivation, herpes simplex virus, and aspergillus infections [6]. In addition, in alemtuzumab treated patients, EBV reactivation has been described and several cases of EBV associated systemic lymphoma have been reported [5, 7].

Our patient developed a cerebral EBV-positive immunodeficiency lymphoma during alemtuzumab treatment. The positive CD23 staining makes a transformation from the known B-CLL a theoretical possibility, but otherwise no clonal relationship between the CLL and NHL were observed. Both in CSF and serum high copy numbers of EBV were demonstrated. Moreover, the EBER staining of the biopsy specimen was positive, identifying the relation with EBV. Of note, in occasional cases of malignant transformation in CLL, EBV has been identified in the higher-grade neoplasm [3]. A retrospective study showed $16 \%$ of 25 patients with malignant transformation of CLL to be EBV-positive, indicating a role for EBV in malignant transformation in leukemia [1]. In our case, PCR EBV and CMV monitoring was not performed during treatment with alemtuzumab, and the ELISA assay for anti-EBV antibodies remained negative. Only when the patient developed severe neurological symptoms the EBV PCR was done which revealed both in serum and CSF the EBV reactivation. PCR techniques detecting EBV have a high sensitivity compared to the detection of antibodies with ELISA and are not influenced by an immunocompromised state.

Because of the increasing use of alemtuzumab and the profound and lasting immunosuppression this drug induces, neurologists should be aware of opportunistic infections including EBV. Regular monitoring of EBV and CMV using PCR is indicated in patients treated with alemtuzumab. If alemtuzumab treated patients develop neurological signs and symptoms, opportunistic infections and EBV induced lymphoma must be considered.

Conflict of interest $\quad$ B. van de Langerijt reports no disclosures; J.K. Doorduijn reports no disclosures; K.H. Lam reports no disclosures; M.J. van den Bent reports no disclosures

Open Access This article is distributed under the terms of the Creative Commons Attribution Noncommercial License which permits any noncommercial use, distribution, and reproduction in any medium, provided the original author(s) and source are credited.

\section{References}

1. Ansell SM, Li CY, Lloyd RV, Phyliky RL (1999) Epstein-Barr virus infection in Richter's transformation. Am J Hematol 60:99-104

2. Bagic A, Lupu VD, Kessler CM, Tornatore C (2007) Isolated Richter's transformation of the brain. J Neurooncol 83:325-328

3. Foon KA, Thiruvengadam R, Saven A, Bernstein ZP, Gale RP (1993) Genetic relatedness of lymphoid malignancies. Transformation of chronic lymphocytic leukemia as a model. Ann Intern Med 119:63-73

4. Hanse MC, Van't Veer MB, van Lom K, Van den Bent MJ (2008) Incidence of central nervous system involvement in chronic lymphocytic leukemia and outcome to treatment. J Neurol 255:828-830

5. Kluin-Nelemans HC, Coenen JL, Boers JE, van Imhoff GW, Rosati S (2008) EBV-positive immunodeficiency lymphoma after alemtuzumab-CHOP therapy for peripheral T-cell lymphoma. Blood 112:1039-1041

6. Martin SI, Marty FM, Fiumara K, Treon SP, Gribben JG, Baden LR (2006) Infectious complications associated with alemtuzumab use for lymphoproliferative disorders. Clin Infect Dis 43:16-24

7. Scheinberg P, Fischer SH, Li L, Nunez O, Wu CO, Sloand EM, Cohen JI, Young NS, John Barrett A (2007) Distinct EBV and CMV reactivation patterns following antibody-based immunosuppressive regimens in patients with severe aplastic anemia. Blood 109:3219-3224 